\title{
Invariant Torus in 3D Lotka-Volterra Systems Appearing After Perturbation of Hopf Center
}

\author{
Marcin Bobieński
}

Received: 7 January 2010 / Accepted: 22 March 2010 / Published online: 9 February 2011

C The Author(s) 2011. This article is published with open access at Springerlink.com

\begin{abstract}
We study a three dimensional Lotka-Volterra systems. In the paper Bobieński and Żołądek (J Ergod Theory Dyn Syst 25:759-791, 2005) four cases of center (i.e. an invariant surface supporting a center) were found. In this paper, we study a codimension 2 component $L V^{H o p f}$ and its versal deformation. We prove that at most one invariant torus may appear. This invariant torus corresponds to the limit cycle bifurcating in the amplitude system.
\end{abstract}

Keywords Lotka-Volterra system $\cdot$ Abelian integrals $\cdot$ Limit cycles

Mathematics Subject Classification (2000) Primary 34C07 · 37C27; Secondary $34 \mathrm{C} 23 \cdot 34 \mathrm{C} 26$

\section{Introduction}

The class of 2-dimensional Lotka-Volterra system is one of the most important and quite well investigated (see $[4,6,8,9]$ ). In particular, the center problem is completely solved. In higher dimension the situation is much more complicated (see [5]). In [2], the center problem for 3-dimensional Lotka-Volterra systems of the general form

Supported by Polish KBN Grant No 2 P03A 01529.

M. Bobieński $(\varangle)$

Institute of Mathematics, Warsaw University,

ul. Banacha 2, 02-097 Warsaw, Poland

e-mail: mbobi@mimuw.edu.pl 


$$
\begin{aligned}
& \dot{x}=x(a y+b z+\lambda u) \\
& \dot{y}=y(c z+d x+\mu u) \\
& \dot{z}=z(e x+f y+v u),
\end{aligned}
$$

(where $u=x+y+z-1$ ) was investigated. We asked about the existence of a 1-dimensional, continuous family of closed orbits. In the 9-dimensional space of systems (1.1), four cases (subvarieties) of center were found. Three of them are explicit in a sense that the center is a consequence of existence of first integral on the invariant surface. The fourth component $L V^{H o p f}$ is quite different. This component forms a codimension 2 subvariety (given by the pair of equation (2.1)) in the parameter space and it corresponds to the following situation. The system (1.1) admits a first integral of Darboux form and a line of critical points. The system (1.1) restricted to a level surface of first integral (invariant surface) admits a unique limit cycle which is a result of classical Hopf bifurcation theorem (changing stability of singular point of planar vector field). These 2-dimensional limit cycles of the restricted system form a 1-parameter family of periodic orbits (center)—see Sect. 2 or the paper [2] for more details.

We consider 2-dimensional, normal deformation of a system $X_{0} \in L V^{H o p f}$ inside systems of (1.1) form. The aim is to investigate the phase portrait of the perturbed system, sufficiently close to the center singular point. Some bifurcations of the system has already been studied in [3]. Appearance of limit cycle or escape of a singular point outside the considered region were proved for a particular system $X_{0}$ from $L V^{H o p f}$. To complete the bifurcation diagram of normal perturbation of $L V^{H o p f}$ it remains to investigate neighborhood of certain parabola. This curve corresponds to the limit cycle bifurcation in amplitude system which means a 2-dimensional invariant torus in 3-dimensional system. In this paper, we investigate this torus bifurcation for a generic point on the stratum $L V^{H o p f}$ of the center variety. The main result is the proof that at most one invariant torus can bifurcate. Moreover, we can control the size of this torus (in terms of perturbation parameters) and so we are able to complete the bifurcation diagram.

\section{Statement of the Results}

We consider the space of three dimensional Lotka-Volterra systems $X$ given by the family (1.1). To avoid the degenerate case, when one can divide out a common linear factor, we assume that at least two of linear cofactors $(a y+b z+\lambda u),(c z+d x+$ $\mu u),(e x+f y+v u)$ are not proportional. Thus, the parameter space is an open dense set in the 9-dimensional vector space with coordinates $(a, b, c, d, e, f, \lambda, \mu, v)$. In [2] four cases of center among these systems were found; the respective subvarieties of the parameter space were denoted $L V_{0}^{\text {plane }}, L V_{1}^{\text {plane }}, L V^{\text {Darb }}, L V^{H o p f}$. First three center cases have similar nature. For example, the $L V_{0}^{\text {plane }}$ case is described by three equations and one inequality in the parameter space

$$
L V_{0}^{\text {plane }}: \quad a+d=b+e=c+f=0, \quad a b d(a+b-d)<0 .
$$


The system (1.1) with above restrictions, $X_{0}$ has the invariant plane $x+y+z=1$ and the restricted system $\left.X_{0}\right|_{x+y+z=1}$ has Darboux type first integral $H=|x|^{-d}|y|^{b}|z|^{a}$. The center in this case is formed by the nest of cycles on the invariant plane. The investigation of the phase portrait of perturbation of any center system from $L V_{0}^{\text {plane }}$ can be performed by standard techniques of perturbations of 2-dimensional (quadratic) integrable systems.

Similar situation occurs in the $L V_{1}^{\text {plane }}$ and $L V^{\text {Darb }}$ cases; the center is a consequence of the existence of the invariant surface (given explicitly) and of the first integral of the restricted system.

The center in the case $L V^{H o p f}$ has quite different nature. The $L V^{H o p f}$ is a codimension 2 variety in the parameter space. It is defined by the following two equations

$$
L V^{H o p f}: \operatorname{det}\left(\begin{array}{lll}
0 & a & b \\
d & 0 & c \\
e & f & 0
\end{array}\right)=0, \quad(\lambda, \mu, v) \perp \operatorname{ker}\left(\begin{array}{lll}
0 & a & b \\
d & 0 & c \\
e & f & 0
\end{array}\right)
$$

and two additional inequalities we describe invariantly below. Let a system $X$ satisfies conditions (2.1). The function $E=|x|^{\alpha}|y|^{\beta}|z|^{\gamma}$, where a vector $(\alpha, \beta, \gamma)$ generates a left kernel of matrix $\left(\begin{array}{lll}0 & a & b \\ d & 0 & c \\ e & f & 0\end{array}\right)$, is a first integral of the system $X$. On the other hand, the system has a line of critical points (zeroes) given by three (linearly dependent) equations $(a y+b z+\lambda u)=0,(c z+d x+\mu u)=0,(e x+f y+v u)=0$. The divergence $\operatorname{div} X$ of the system $X$ is a linear function. The first inequality of the variety $L V^{H o p f}$ states that $\operatorname{div} X$ is not constant on the line $l$. Under this condition the non-constant linear function $\left.(\operatorname{div} X)\right|_{l}$ vanishes in precisely one point $p_{*}$. Eigenvalues of the linearization of $X$ at $p_{*} \mathrm{~d} X\left(p_{*}\right)$ are so $0, \pm \lambda_{*}$. The second inequality reads: $\left(\lambda_{*}\right)^{2}<0$.

Remark 2.1 A pair of inequalities discussed above can be interpreted in the following way. Let $n$ generate a right kernel of the matrix $A=\left(\begin{array}{lll}0 & a & b \\ d & 0 & c \\ e & f & 0\end{array}\right)$. The first inequality is equivalent to $\nabla_{n} \operatorname{div} X \neq 0$ (the left hand side is a constant). Then the characteristic polynomial of derivative $\mathrm{d} X$ at the point $p_{*}$ reads $\operatorname{det}\left(\mathrm{d} X\left(p_{*}\right)-k I\right)=-k^{3}+k^{2}-D k$. The second condition states that $D>0$.

In [2] it is proved that a Lotka-Volterra system $X$ satisfying the above conditions provides a center, i.e. one parameter family of closed orbits of $X$. Let me shortly recall the argument. The system restricted to the invariant level curves of the first integral $E=|x|^{\alpha}|y|^{\beta}|z|^{\gamma}$ has a singular point corresponding to the intersection of $E^{-1}(h)$ and the line $l$. The stability of the singular point of the planar restricted system $X_{E^{-1}(h)}$ changes precisely as the value of parameter $h$ passes $E\left(p_{*}\right)$. The nondegenerate Hopf bifurcation generates a 2-dimensional limit cycles of system $X$ restricted to level curves of $E$. They form a center, provided we are sufficiently close to the singular point $p_{*}$.

The main aim of this paper is to investigate the bifurcation diagram of a system from the $L V^{H o p f}$ perturbed in normal directions. Since $L V^{H o p f}$ is a codimension 2 subvariety the normal perturbation is of the form 


$$
X_{\varepsilon}=X_{0}+\varepsilon_{1} P+\varepsilon_{2} Q,
$$

where $X_{0} \in L V^{H o p f}$ and (quadratic) systems $P, Q$ generate perturbations in normal directions. We adapt coordinates to the dynamics of the unperturbed system $X_{0}$. Let $v=E$ be the first integral of $X_{0}$. The linearization matrix $\mathrm{d} X_{0}\left(p_{*}\right)$ around $p_{*}$ have eigenvalues $(0,+i \theta,-i \theta)$. Note, that eigenvalue 0 corresponds to the direction of gradient of the first integral $E$. Let $w$ be a complex coordinate related to the eigenvalue $+i \theta$. Finally, we change coordinates $(v, w)$ to transform the system $X_{0}$ into the Poincaré-Dulac normal form [1] up to degree 3 . The system $X_{0}$ in new coordinates $(v, w)$ reads as follows

$$
X_{0}=\left\{\begin{array}{l}
\dot{w}=w\left(i \theta+A v+B v^{2}+C|w|^{2}\right)+\text { h.o.t. } \\
\dot{v}=0
\end{array}\right.
$$

We apply the same idea to the perturbed system $X_{\varepsilon}$ :

$$
X_{\varepsilon}=\left\{\begin{array}{l}
\dot{w}=w\left(i \theta+t_{\varepsilon}+A_{\varepsilon} v+B_{\varepsilon} v^{2}+C_{\varepsilon}|w|^{2}\right)+R_{w}\left(\varepsilon_{1}, \varepsilon_{2}, v, w, \bar{w}\right) \\
\dot{v}=f_{\varepsilon}+g_{\varepsilon} v+h_{\varepsilon}|w|^{2}+k_{\varepsilon} v^{2}+R_{v}\left(\varepsilon_{1}, \varepsilon_{2}, v, w, \bar{w}\right)
\end{array}\right.
$$

where $t_{\varepsilon}=t_{1} \varepsilon_{1}+t_{2} \varepsilon_{2}, a_{\varepsilon}=a_{1} \varepsilon_{1}+a_{2} \varepsilon_{2}$, etc. The expression $R_{w}$ represents all terms of degree $\geq 4$ and terms of order $O\left(|\varepsilon|^{2}\right)$; the element $R_{v}$ represents all terms of degree $\geq 3$ and terms of order $O\left(|\varepsilon|^{2}\right)$. Qualitative properties of the above system are determined by 2 -dimensional amplitude system, i.e. the system (2.4) in the variable $\rho=|w|^{2}$. The amplitude system is following

$$
\begin{aligned}
\dot{\rho} & =2 \rho\left(\hat{t}_{\varepsilon}+\hat{A}_{\varepsilon} v+\hat{C}_{\varepsilon} \rho\right)+\ldots \\
\dot{v} & =f_{\varepsilon}+g_{\varepsilon} v+h_{\varepsilon} \rho+\ldots
\end{aligned}
$$

where $\hat{A}_{\varepsilon}=\operatorname{Re} A_{\varepsilon}, \hat{t_{\varepsilon}}=\operatorname{Re}\left(t_{\varepsilon}\right)$, etc. Note that the coefficients $\hat{t_{\varepsilon}}, f_{\varepsilon}, g_{\varepsilon}, h_{\varepsilon}, \ldots$ (all except the capital ones) vanish for $\varepsilon_{1}=\varepsilon_{2}=0$; it follows directly from the (2.3) form of the unperturbed system $X_{0}$.

It turns out that generically (the genericity conditions precisely described in Theorem 2.5 below) the global properties of the phase portrait of the amplitude system (2.5) is fully determined by leading terms, except the (smooth) curve corresponding to the center. To simplify notation we denote

$$
\Delta=\hat{C}_{\varepsilon} g_{\varepsilon}-\hat{A}_{\varepsilon} h_{\varepsilon} \quad F_{C e n}:=2\left(\hat{A}_{\varepsilon} f_{\varepsilon}-\hat{t}_{\varepsilon} g_{\varepsilon}\right) \hat{C}_{\varepsilon}+\Delta g_{\varepsilon} .
$$

Let $X_{\rho}^{0}$ be the leading part of the the amplitude system (2.5), i.e. the system (2.5) with dots skipped.

Proposition 2.2 Assume that $\hat{A}_{0} \neq 0, \hat{C}_{0} \neq 0,\left.\frac{\partial\left(\Delta, f_{\varepsilon}\right)}{\partial\left(\varepsilon_{1}, \varepsilon_{2}\right)}\right|_{\varepsilon=0} \neq 0,\left.\frac{\partial\left(g_{\varepsilon}, f_{\varepsilon}\right)}{\partial\left(\varepsilon_{1}, \varepsilon_{2}\right)}\right|_{\varepsilon=0} \neq 0$. Then foror $\left(\varepsilon_{1}, \varepsilon_{2}\right)$ sufficiently close to $(0,0)$ the system $X_{\rho}^{0}$ has a center at the point 
$\left(\rho_{*}, v_{*}\right)=\left(\frac{\hat{A}_{\varepsilon} f_{\varepsilon}-g_{\varepsilon} \hat{t}_{\varepsilon}}{\Delta}, \frac{\hat{C}_{\varepsilon} f_{\varepsilon}-h_{\varepsilon} \hat{t}_{\varepsilon}}{\Delta}\right)$ if and only if the following conditions are satisfied

$$
F_{C e n}=0,\left.\quad \hat{C}_{0}\left(\frac{\partial\left(\Delta, f_{\varepsilon}\right)}{\partial\left(\varepsilon_{1}, \varepsilon_{2}\right)} \frac{\partial\left(g_{\varepsilon}, f_{\varepsilon}\right)}{\partial\left(\varepsilon_{1}, \varepsilon_{2}\right)}\right)\right|_{\varepsilon=0}<0 .
$$

On the positive part of the center curve $\left(F_{C e n}=0, \Delta>0\right)$ the coordinate of the center $\rho_{*}$ is positive.

It was proved in [3] that outside a narrow neighborhood of the center curve the bifurcational diagram of the amplitude system is fully determined by the qualitative properties of the leading part $X_{\rho}^{0}$. Near center the situation is unstable so one must take into account higher order terms; one obtains the following perturbation of a quadratic integrable system:

$$
\begin{aligned}
& \dot{\rho}=2 \rho\left(\hat{t}_{\varepsilon}+\hat{A}_{\varepsilon} v+\hat{C}_{\varepsilon} \rho+\widetilde{P}_{\varepsilon}\right) \\
& \dot{v}=f_{\varepsilon}+g_{\varepsilon} v+h_{\varepsilon} \rho+\widetilde{Q}_{\varepsilon},
\end{aligned}
$$

where $\widetilde{P}_{\varepsilon}, \widetilde{Q}_{\varepsilon}$ are quadratic polynomials in $\rho, v$. System $(2.7)$ is a perturbation of the integrable system and so it generates a pseudo-Abelian integral in a usual way. Investigation of this situation was a main aim of this paper. The main result of the paper is the following theorem

Theorem 2.3 Assume that $\hat{A}_{0} \neq 0,\left.\hat{C}_{0}\left(\frac{\partial\left(\Delta, f_{\varepsilon}\right)}{\partial\left(\varepsilon_{1}, \varepsilon_{2}\right)} \frac{\partial\left(g_{\varepsilon}, f_{\varepsilon}\right)}{\partial\left(\varepsilon_{1}, \varepsilon_{2}\right)}\right)\right|_{\varepsilon=0}<0$. The (germ of) curve

$$
\mathrm{Cen}^{+}=\left\{\left(\varepsilon_{1}, \varepsilon_{2}\right): F_{C e n}\left(\varepsilon_{1}, \varepsilon_{2}\right)=0, \Delta\left(\varepsilon_{1}, \varepsilon_{2}\right)>0\right\}
$$

on the $\left(\varepsilon_{1}, \varepsilon_{2}\right)$ plane is smooth and the leading amplitude system $X_{\rho}^{0}$ has a center on it. The pseudo-Abelian integral generated by the amplitude system (2.7) does not vanish identically and it has at most one simple zero. The value of this simple zero tends to infinity along a (germ of) smooth curve $T_{\infty}$ on the $\left(\varepsilon_{1}, \varepsilon_{2}\right)$ plane. The order of tangency at $(0,0)$ of curves $\mathrm{Cen}^{+}$and $T_{\infty}$ is equal 3.

Under additional genericity condition, which guarantee that certain coefficient in the presentation of the pseudo-Abelian integral does not vanish, one can fully control the bifurcation diagram of the amplitude system in a neighborhood of the center curve $\mathrm{Cen}^{+}$.

Theorem 2.4 Assume that the normal deformation $X_{\varepsilon}$ of a system $X_{0} \in L V^{H o p f}$ satisfies assumptions of Theorem 2.3. Assume that $C_{\varepsilon_{1}} \neq 0$, where $C_{\varepsilon_{1}}$ is the coefficient in the pseudo-Abelian presentation (3.8) (genericity condition). Then there exist two smooth curves $\mathrm{Cen}^{+}, T_{\infty}$ on the $\left(\varepsilon_{1}, \varepsilon_{2}\right)$ plane which are bifurcational for the system $X_{\varepsilon}$. Crossing the line Cen ${ }^{+}$generates unique invariant torus $T$ which escapes to infinity (outside the region which is under control) along the line $T_{\infty}$. These curves are tangent at $\varepsilon=0$ up to $O\left(|\varepsilon|^{3}\right)$ order. 
Using Theorem 2.4 and results of [3], one can draw the full bifurcational diagram in a generic case. To simplify picture we will draw the diagram for the 2-dimensional amplitude system.

Theorem 2.5 Assume that $\hat{A}_{0} \neq 0, \hat{C}_{0} \neq 0,\left.\frac{\partial\left(\Delta, f_{\varepsilon}\right)}{\partial\left(\varepsilon_{1}, \varepsilon_{2}\right)}\right|_{\varepsilon=0} \neq 0,\left.\frac{\partial\left(g_{\varepsilon}, f_{\varepsilon}\right)}{\partial\left(\varepsilon_{1}, \varepsilon_{2}\right)}\right|_{\varepsilon=0} \neq 0$. There are 4 germs of smooth curves on the $\varepsilon_{1}, \varepsilon_{2}$ plane:

$$
\begin{array}{rlrl}
p_{\infty}: & \Delta & =0, \\
\gamma_{\infty}: & g_{\varepsilon} & =0, \\
H: & \hat{A}_{\varepsilon} f_{\varepsilon}-\hat{t}_{\varepsilon} g_{\varepsilon} & =0, \\
& \text { Cen }^{+}: & F_{C e n} & =0, \quad \Delta>0 .
\end{array}
$$

The curves $p_{\infty}, \gamma_{\infty}, H$ are pairwise transversal and $C e n^{0}$ is tangent to $H$. The curves $p_{\infty}, \gamma_{\infty}, H$ are bifurcational for the amplitude system.

1. If $\left.\hat{C}_{0}\left(\frac{\partial\left(\Delta, f_{\varepsilon}\right)}{\partial\left(\varepsilon_{1}, \varepsilon_{2}\right)} \frac{\partial\left(g_{\varepsilon}, f_{\varepsilon}\right)}{\partial\left(\varepsilon_{1}, \varepsilon_{2}\right)}\right)\right|_{\varepsilon=0}<0$ then curves $C e n^{+}$and $T_{\infty}$ are also bifurcational; the invariant torus $T$ is born on the line $\mathrm{Cen}^{+}$and it escapes to infinity along $T_{\infty}$. The bifurcational diagram in this case is drawn on Fig. 1

2. If $\left.\hat{C}_{0}\left(\frac{\partial\left(\Delta, f_{\varepsilon}\right)}{\partial\left(\varepsilon_{1}, \varepsilon_{2}\right)} \frac{\partial\left(g_{\varepsilon}, f_{\varepsilon}\right)}{\partial\left(\varepsilon_{1}, \varepsilon_{2}\right)}\right)\right|_{\varepsilon=0}>0$ then curves $C_{e n}{ }^{+}$and $T_{\infty}$ are not bifurcational and the bifurcational diagram simplifies to Fig. 2.

Singular points $p$ and $\gamma$ of the amplitude system correspond to the singular point and the limit cycle of the three dimensional system $X_{\varepsilon}$, respectively.

Remark 2.6 The above theorems confirm predictions posted in [3] and recently also in [7] about the bifurcation diagram of the normal deformation of the $L V^{H o p f}$ component.

\subsection{Further Tasks}

Theorem 2.5 describes the bifurcational diagram of the perturbation of the 3-dimensional Lotka-Volterra systems under genericity assumptions. The first task which is worth to study is to understand the geometric nature of the genericity restrictions in terms of the original, 3-dimensional Lotka-Volterra systems. In particular, it is interesting to investigate the case when the coefficient $C_{\varepsilon_{1}}$ in the presentation of pseudoAbelian integral vanishes. In this case, one should take into account higher order terms in the Dulac normal form of the amplitude system.

Another interesting investigation task, pointed in [3], is to investigate the dynamics of the system restricted to the invariant torus.

\section{Analysis of the Pseudo-Abelian Integral Generated by the Amplitude System}

The aim of this section is to study the properties of the amplitude system (2.7). We focus on sufficiently small neighborhood of the origin. The leading term of the amplitude system is a quadratic system $X_{\rho}^{0}((2.5)$ with dots skipped). The quadratic system $X_{\rho}^{0}$ has two critical points: 


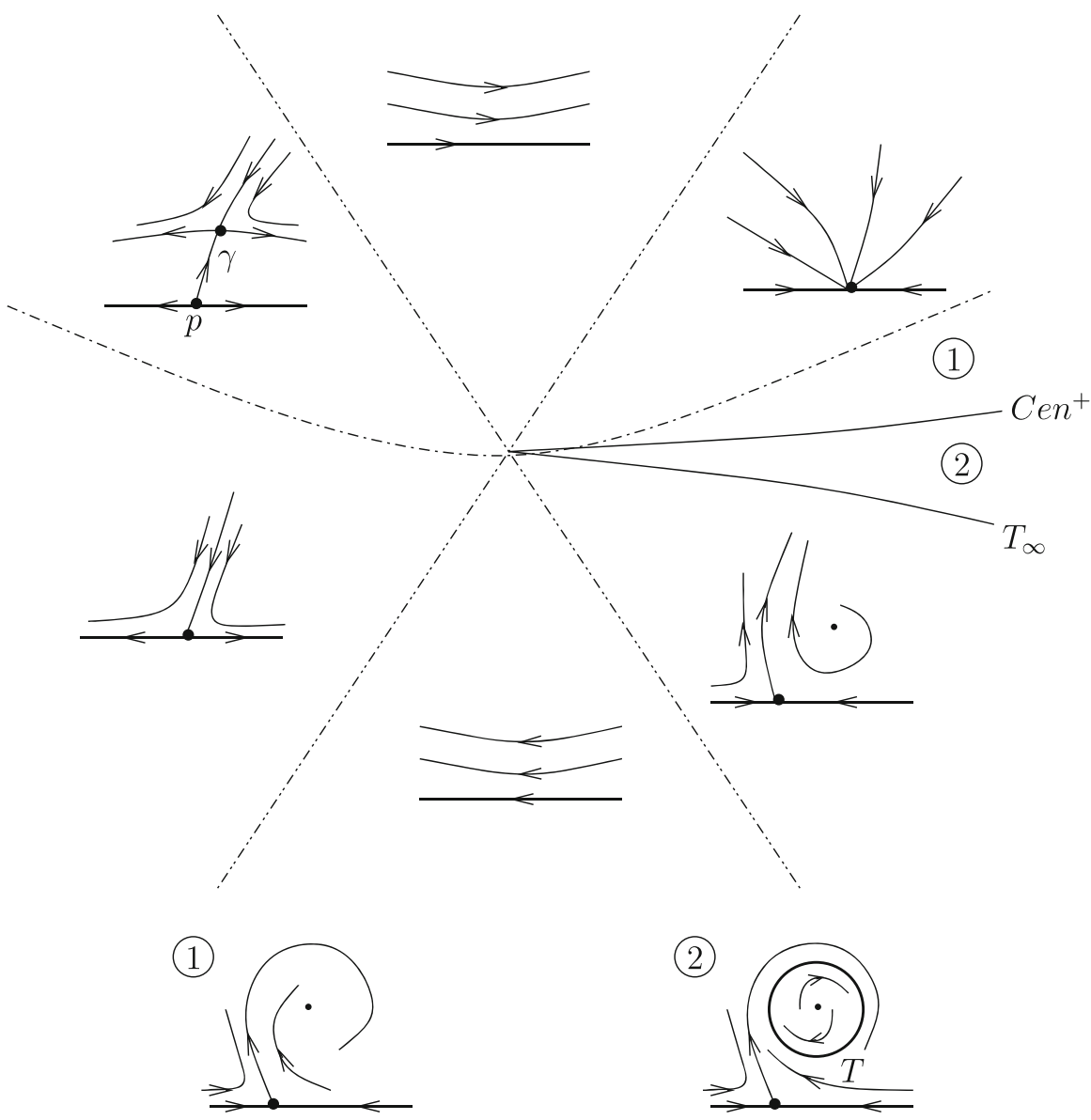

Fig. 1 Bifurcation diagram of the amplitude system in case (1)

$$
p=\left(\rho=0, v_{0}=-\frac{f_{\varepsilon}}{g_{\varepsilon}}\right), \quad \gamma=\left(\rho_{*}=\frac{\hat{A}_{\varepsilon} f_{\varepsilon}-g_{\varepsilon} \hat{t}_{\varepsilon}}{\Delta}, v_{*}=\frac{\hat{C}_{\varepsilon} f_{\varepsilon}-h_{\varepsilon} \hat{t}_{\varepsilon}}{\Delta}\right)
$$

where $\Delta=\hat{C}_{\varepsilon} g_{\varepsilon}-\hat{A}_{\varepsilon} h_{\varepsilon}$. Note that $p$ corresponds to a critical point of the 3-dimensional system and $\gamma$ corresponds to a limit cycle.

According to the well known result for quadratic planar vector field [10], the system $X_{\rho}^{0}$ has a center in $\gamma$ if and only if $\operatorname{tr}\left(L_{*}\right)=0$ and $\operatorname{det}\left(L_{*}\right)>0$, where $L_{*}$ is a linearization matrix in point $\gamma$. Thus, the center condition reads

$$
2 \rho_{*} \hat{C}_{\varepsilon}+\delta=0, \quad 2 \rho_{*} \Delta>0 .
$$

Using the first equation, one can transform the second inequality to $-\frac{g_{\varepsilon} \Delta}{\hat{C}_{\varepsilon}}>0$ which must be satisfied on the curve $2 \rho_{*} \hat{C}_{\varepsilon}+g_{\varepsilon}=0$. Note that all coefficients denoted by small letters (e.g. $f_{\varepsilon}, g_{\varepsilon}, h_{\varepsilon}, \ldots$ ) vanishes for $\varepsilon=0$. Thus, the equation 
Fig. 2 Bifurcation diagram of the amplitude system in case (2)

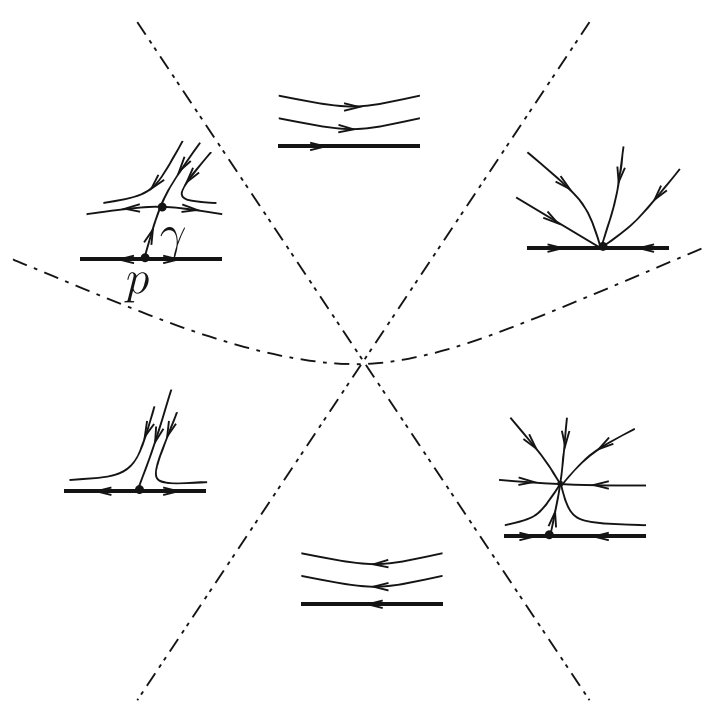

$2 \rho_{*} \hat{C}_{\varepsilon}+g_{\varepsilon}=0$ has the form $f_{\varepsilon}=O\left(\varepsilon^{2}\right)$. By the genericity assumption $\left.\frac{\partial\left(\Delta, f_{\varepsilon}\right)}{\partial\left(\varepsilon_{1}, \varepsilon_{2}\right)}\right|_{\varepsilon=0} \neq$ 0 the following transformation

$$
\left(\varepsilon_{1}, \varepsilon_{2}\right) \mapsto\left(\Delta, f_{\varepsilon}\right)
$$

is a germ of diffeomorphism. In the new parametrization provided by $\left(\Delta, f_{\varepsilon}\right)$, the inequality $-\frac{g_{\varepsilon} \Delta}{\hat{C}_{\varepsilon}}>0$ is equivalent to $\hat{C}_{0}\left(\frac{\partial g}{\partial \Delta}\right)(0)<0$; the latter is equivalent to (2.6). Moreover, since $\rho_{*}=-\frac{g_{\varepsilon}}{2 \hat{C}_{\varepsilon}}$, the coordinate $\rho_{*}$ is positive provided $\Delta>0$ (center equation assumed).

This finishes proof of Proposition 2.2

Due to the assumptions $\left.\frac{\partial\left(\Delta, f_{\varepsilon}\right)}{\partial\left(\varepsilon_{1}, \varepsilon_{2}\right)}\right|_{\varepsilon=0} \neq 0, \hat{A}_{0} \neq 0, \hat{C}_{0} \neq 0$ the following map is a germ of diffeomorphism defined on the neighborhood of $(0,0)$

$$
\left(\tilde{\varepsilon}_{1}, \tilde{\varepsilon}_{2}\right)=\left(\Delta, F_{C e n}\right)=\left(\Delta, 2\left(\hat{A}_{\varepsilon} f_{\varepsilon}-\hat{t}_{\varepsilon} g_{\varepsilon}\right) \hat{C}_{\varepsilon}+\Delta g_{\varepsilon}\right),
$$

where $\Delta=\hat{C}_{\varepsilon} g_{\varepsilon}-\hat{A}_{\varepsilon} h_{\varepsilon}$. Indeed, the only term of the function $F_{C e n}$ linear in $\varepsilon$ is $2 \hat{A}_{\varepsilon} \hat{C}_{\varepsilon} f_{\varepsilon}$. Until the end of the paper we will work with $\tilde{\varepsilon}=\left(\tilde{\varepsilon}_{1}, \tilde{\varepsilon}_{2}\right)$. To simplify notation we will skip $\sim$ index above $\varepsilon$. Note that

$$
f_{\varepsilon}=f_{2} \varepsilon_{2}+O\left(\varepsilon^{2}\right), \quad f_{2} \neq 0
$$

Since we want to investigate a narrow neighborhood of the center line $\left(\varepsilon_{2}=0\right)$ in the parameter space and a neighborhood of the origin in the phase space, we make the following rescaling 


$$
\varepsilon_{2}=\delta \varepsilon_{1}^{2}, \quad \rho=\varepsilon_{1} R, \quad v=\varepsilon_{1} W .
$$

We will work with system (2.7) in the Pfaff form $\omega=\dot{\rho} \mathrm{d} v-\dot{v} \mathrm{~d} \rho$. In rescaled variables the one form $\omega$ up to terms linear in $\left(\varepsilon_{1}, \delta\right)$ reads

$$
\begin{aligned}
\omega_{\varepsilon_{1}, \delta}=\frac{\omega}{\varepsilon_{1}{ }^{3}}= & 2 R\left(t_{\varepsilon_{1}}+A_{\varepsilon_{1}} W+C_{\varepsilon_{1}} R\right) \mathrm{d} W+\left(f_{\varepsilon_{1}}+g_{\varepsilon_{1}} W+h_{\varepsilon_{1}} R\right) \mathrm{d} R \\
& +\delta f_{2} \mathrm{~d} R+\varepsilon_{1}(2 R P(R, W) \mathrm{d} W-Q(R, W) \mathrm{d} R) .
\end{aligned}
$$

Using pull-back of the following affine transformation (a parameter $\alpha$ which will be fixed later)

$$
(R, W)=\Phi\left(R_{1}, W_{1}\right)=\left(-\frac{g_{\varepsilon_{1}}}{2 C_{\varepsilon_{1}}}\left(1+R_{1}\right), \quad \frac{g_{\varepsilon_{1}} \alpha}{2 A_{\varepsilon_{1}}}\left(\alpha W_{1}+\alpha^{-1} R_{1}\right)+\frac{g_{\varepsilon_{1}}-2 t_{\varepsilon_{1}}}{2 A_{\varepsilon_{1}}}\right)
$$

we obtain (index 1 at $(R, W)$ variables omitted)

$$
\begin{aligned}
\Phi^{*} \omega_{\varepsilon_{1}, \delta}= & \text { const }\left(\alpha W(1+R) \mathrm{d} W+\alpha^{-1} R\left(W-\frac{\Delta_{\varepsilon_{1}}}{g_{\varepsilon_{1}} C_{\varepsilon_{1}} \alpha^{2}}\right) \mathrm{d} R\right) \\
& +\delta \tilde{f}_{2} \mathrm{~d} R+\varepsilon_{1}\left(\widetilde{Q}(R, W) \mathrm{d} R+(1+R) \widetilde{P}_{1}\left(\alpha \mathrm{d} W+\alpha^{-1} \mathrm{~d} R\right)\right)
\end{aligned}
$$

where $\Delta_{\varepsilon_{1}}=C_{\varepsilon_{1}} g_{\varepsilon_{1}}-A_{\varepsilon_{1}} h_{\varepsilon_{1}}$ and $\tilde{f}_{2} \neq 0$. The leading part of system (3.5) $\omega_{C e n}$ provides a center at the point $(R, W)=(0,0)$ if and only if $\frac{\Delta_{\varepsilon_{1}}}{g_{\varepsilon_{1}} C_{\varepsilon_{1}}}\left(\varepsilon_{1}=0\right)<0$; the latter condition is equivalent to the inequality (2.6). Assuming the condition is satisfied, we define

$$
\alpha=\sqrt{-\frac{\Delta}{g_{\varepsilon_{1}} C_{\varepsilon_{1}}}} .
$$

It transforms the integrable leading part $\omega_{\text {Cen }}$ to the following canonical form

$$
\omega_{C e n}=\alpha W(1+R) \mathrm{d} W+\alpha^{-1} R(1+W) \mathrm{d} R .
$$

Dividing $\omega_{C e n}$ by the integrating factor $M=(1+R)(1+W)$ gives derivative of the following function which is a first integral of foliation $\omega_{C e n}$

$$
H(R, W)=\alpha(W-\log (1+W))+\alpha^{-1}(R-\log (1+R)) .
$$

The linearization of the Poincaré map is given by the following pseudo-Abelian integral

$$
\begin{aligned}
I(h)= & \delta \tilde{f}_{2} \int_{\gamma_{h}} \frac{\mathrm{d} R}{(1+R)(1+W)} \\
& +\varepsilon_{1} \int_{\gamma_{h}}\left(\frac{\widetilde{Q}_{1} \mathrm{~d} R}{(1+R)(1+W)}+\frac{\widetilde{P}_{1}}{1+W}\left(\alpha \mathrm{d} W+\alpha^{-1} \mathrm{~d} R\right)\right),
\end{aligned}
$$

where $\gamma_{h} \subset H^{-1}(h)$ is a real oval. 
We define the following base integrals. Below $\Gamma_{h}$ denotes the compact subset of $\mathbb{R}^{2}$ bounded by the oval $\gamma_{h}=H^{-1}(h)$, i.e. $\partial \Gamma_{h}=\gamma_{h}$.

$$
\begin{aligned}
& S(h)=\int_{\gamma_{h}} \alpha^{-1} R\left(\alpha \mathrm{d} W+\alpha^{-1} \mathrm{~d} R\right)=\int_{\Gamma_{h}} 1=\operatorname{Area}\left(\Gamma_{h}\right), \\
& J(h)=\int_{\gamma_{h}} \frac{\mathrm{d} R}{1+W}=\int_{\Gamma_{h}} \frac{1}{(1+W)^{2}} .
\end{aligned}
$$

Integrals $J, S$ generate the space of pseudo-Abelian integrals (3.6) (for quadratic $P, Q)$.

Proposition 3.1 The pseudo-Abelian integral I $(h)$ has the following presentation

$$
I(h)=\delta C_{\delta} J+\varepsilon_{1} C_{\varepsilon_{1}}(S-J)
$$

Moreover, $C_{\delta} \neq 0$.

Remark 3.2 Note that the amplitude system (2.7) was derived form 3-dimensional Lotka-Volterra systems. Since the $L V^{H o p f}$ center subspace forms a codimension two subvariety, it is natural to expect that the space of pseudo-Abelian integrals will be generated by two elements (perturbation in directions tangent to $L V^{H o p f}$ should generate zero integral). Details of proof of Proposition 3.1 will be given later.

The proof of Theorem 2.4 is based on the monotonicity of the quotient $\frac{\mathrm{J}}{\mathrm{S}}$.

Proposition 3.3 Assume that $\alpha>0$. Then the quotient of pseudo-Abelian integrals $G(h)=\frac{J}{S}$ is strictly increasing and $\lim _{h \rightarrow 0^{+}} G(h)=1$.

Proof of Theorem 2.4 The non vanishing of the pseudo-Abelian integral $I(h)$ is a consequence of condition $C_{\delta} \neq 0$ (proposition 3.1). If $C_{\varepsilon_{1}}=0$, then the integral $I$ is proportional to function $J$ which does not vanish.

If $C_{\varepsilon_{1}} \neq 0$, then the integral $I(h)$ vanishes in a point $h_{T}$ if and only if

$$
\frac{J}{S}\left(h_{T}\right)=\frac{1}{1-\tilde{C}\left(\delta / \varepsilon_{1}\right)} .
$$

By Proposition 3.3, the function $G=\frac{J}{S}$ is strictly increasing and $\geq 1$. Thus, the unique zero $h_{T}$ exists provided $\frac{\delta}{\varepsilon_{1}} \in(0, M)$ for certain $M$. If the quotient $C_{\delta} / C_{\varepsilon_{1}}$ is negative the same is true for negative $M$. This finishes the proof.

The behaviour of the pseudo-Abelian integral $I(h)$ described in Theorem 2.4 determines the qualitative properties of the amplitude system (2.7) in the neighborhood of the center curve $\mathrm{Cen}^{+}$. Outside this region the properties are ruled by leading quadratic system $X_{\rho}^{0}$ (2.5); the latter one was already investigated in [3]. Summing up these results one can draw the bifurcation diagram of the amplitude system in the generic case. This proves Theorem 2.5. 
Proof of Proposition 3.1. The proof is rather technical. The expectation of two element basis was explained in Remark 3.2.

We have the following identity

$$
\frac{\mathrm{d} R}{(1+R)(1+W)}-\frac{\mathrm{d} R}{1+W}=\alpha^{2} \mathrm{~d}\left(\frac{1+(1+W) \log (1+W)}{1+W}\right)-\frac{\alpha}{(1+W)} \mathrm{d} H
$$

and so

$$
\int_{\gamma_{h}} \frac{\mathrm{d} R}{(1+R)(1+W)}=J
$$

It proves that $C_{\delta}=\tilde{f}_{2} \neq 0$.

In completely analogous way, we derive formula for integral proportional to $\varepsilon_{1}$. By direct calculation we show that for arbitrary homogeneous quadratic polynomials $\widetilde{P}_{1}, \widetilde{Q}_{1}$ there exists a polynomial $K(R, W)$ of degree 4 and constants $\beta$ and $C_{\varepsilon_{1}}$ such that

$$
\begin{aligned}
& \frac{\widetilde{Q}_{1} \mathrm{~d} R}{(1+R)(1+W)}+\frac{\widetilde{P}_{1}}{1+W}\left(\alpha \mathrm{d} W+\alpha^{-1} \mathrm{~d} R\right)=c_{\varepsilon_{1}}\left(R\left(\mathrm{~d} W+\alpha^{-2} \mathrm{~d} R\right)-\frac{\mathrm{d} R}{1+W}\right) \\
& +\mathrm{d}\left(\beta \log (1+W)+\frac{1}{(1+R)(1+W)} K(R, W)\right)+(\ldots) \mathrm{d} H .
\end{aligned}
$$

Note that polynomials $\widetilde{P}_{1}, \widetilde{Q}_{1}$ of degree $\leq 1$ generate integral proportional to $J$.

Proof of Proposition 3.3 The following relation holds

$$
J^{\prime}=\alpha^{-2} J+S^{\prime}
$$

Indeed, we have

$$
J^{\prime}-S^{\prime}=\int_{\gamma_{h}}\left(\frac{1}{(1+W)^{2}}-1\right) \frac{\mathrm{d} W}{\partial_{W} H}=\alpha^{-2} \int_{\gamma_{h}} \frac{-2-R}{1+R} \mathrm{~d} W=\alpha^{-2} \int_{\gamma_{h}} \frac{\mathrm{d} R \wedge \mathrm{d} W}{(1+W)^{2}}=\alpha^{-2} J
$$

Now we use the following technical fact

Proposition 3.4 The function $\log S$ is concave.

Remark 3.5 The above proposition remains valid for any Hamiltonian function of the form $H(R, W)=f(W)+a f(R)$, where $a>0$ and $f(R)$ is a convex function with minimum at $R=0$. Actually we prove this more general statement.

Denote $G(h)=J(h) / S(h)$. It represents average value of $(1+W)^{-2}$ on on $\Gamma_{h}$. As $h \rightarrow 0^{+}$, the loop $\gamma_{h}$ shrinks to a center $(0,0)$ and so the quotient $G$ tends to 1 . Equation (3.9) implies the following equation for $G$

$$
G^{\prime}=G\left(\alpha^{-2}-\frac{S^{\prime}}{S}\right)+\frac{S^{\prime}}{S}
$$


Fig. 3 The equation for $G(h)$
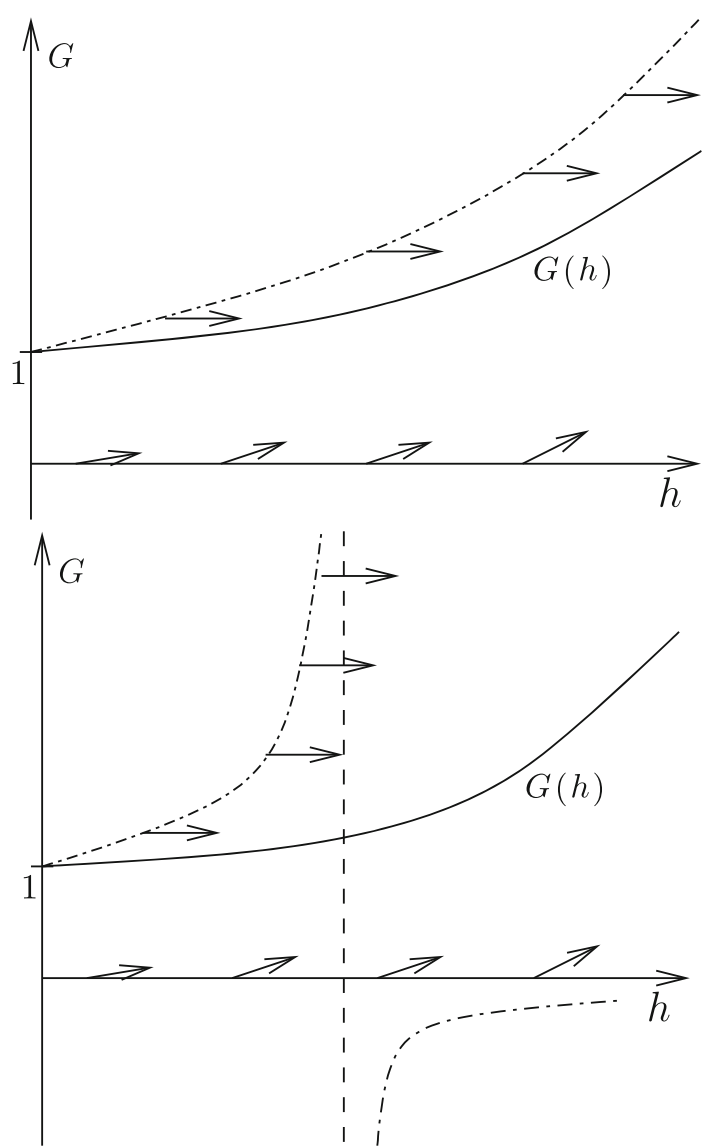

The right hand side of the above equation vanishes on the curve $\widetilde{G}(h)=\frac{S^{\prime} / S}{S^{\prime} / S-1}$. By Proposition 3.4, the curve $\widetilde{G}$ is increasing. Thus, if we present a differential equation for $G(h)$ on the $(G, h)$ plane, it takes one of two possible forms presented on Fig. 3 below (the graph of $\widetilde{G}$ is marked by doted line). In both cases, the solution $G(h)$ can not leave the region bordered by $G=0$ and the graph of $\widetilde{G}$. The right hand side of (3.10) is positive there and so $G$ is strictly increasing. This finishes proof of proposition 3.3.

Proof of Proposition 3.4 Denote $f(R)=R-\log (1+R), a=\alpha^{2}$. We observe that the function $f(R)$ is convex and has a local (and so global) minimum at $R=0$ (see Remark 3.5 above).

We define a function $d(h), h \geq 0$ which is the length of preimage $f^{-1}([0, h])$, i.e. $d(h)=\mu^{1}\left(f^{-1}([0, h])\right)$. The function $d$ is increasing, concave.

The following formulas for the area function $S$ and its derivatives are obtained by a direct calculations 


$$
\begin{aligned}
S & =\int_{0}^{h} d((h-t) / a) d^{\prime}(t) \mathrm{d} t \\
S^{\prime} & =a^{-1} \int_{0}^{h} d^{\prime}((h-t) / a) d^{\prime}(t) \mathrm{d} t, \\
S^{\prime \prime} & =a^{-1} d^{\prime}(h / a) d^{\prime}(h)+a^{-1} \int_{0}^{h}\left[d^{\prime}((h-t) / a)-d^{\prime}(h / a)\right] d^{\prime \prime}(t) \mathrm{d} t .
\end{aligned}
$$

Using formulas (3.11) we obtain the following integral formula for $(\log S)^{\prime \prime}$.

$$
\begin{aligned}
a^{2} S^{2}(\log S)^{\prime \prime}= & a \int_{0}^{h}\left[d^{\prime}((h-t) / a)-d^{\prime}(h / a)\right] d^{\prime \prime}(t) \mathrm{d} t+a d^{\prime}(h / a) d^{\prime}(h) \\
& \times \int_{0}^{h} d((h-t) / a) d^{\prime}(t) \mathrm{d} t-\left(\int_{0}^{h} d^{\prime}((h-t) / a) d^{\prime}(t) \mathrm{d} t\right)^{2} .
\end{aligned}
$$

The first integral is negative, since $d^{\prime \prime}(t)<0$ and $\left[d^{\prime}((h-t) / a)-d^{\prime}(h / a)\right]>0$, by concavity of $d$. The difference of the next two terms is also negative by the following inequalities; they follow the fact that $d^{\prime}$ (respectively $d$ ) is decreasing (respectively increasing) function.

$$
\begin{aligned}
\int_{0}^{h} a^{-1} d^{\prime}((h-t) / a) \frac{d^{\prime}(t)}{d^{\prime}(h)} \mathrm{d} t & \geq \int_{0}^{h} a^{-1} d^{\prime}((h-t) / a) \mathrm{d} t=d(h / a), \\
\int_{0}^{h} \frac{d^{\prime}((h-t) / a)}{d^{\prime}(h / a)} d^{\prime}(t) \mathrm{d} t & \geq \int_{0}^{h} d^{\prime}(t) \mathrm{d} t=d(h), \\
\int_{0}^{h} \frac{d((h-t) / a)}{d(h / a)} d^{\prime}(t) \mathrm{d} t & \leq \int_{0}^{h} d^{\prime}(t) \mathrm{d} t=d(h) .
\end{aligned}
$$

So, $(\log S)^{\prime \prime} \leq 0$ and the proposition 3.4 follows.

Remark 3.6 The above theorems confirm predictions posted in [3] about the bifurcation diagram of the normal deformation of the $L V^{H o p f}$ component.

Acknowledgments The preliminary version of the paper was written while the author was visiting the Laboratorie Emile Picard, Université Paul Sabatier. The author thanks the University for hospitality. The author thanks H. Żołądek for helpful discussions. The author thanks the referee for his helpful suggestions concerning the presentation of this paper. 
Open Access This article is distributed under the terms of the Creative Commons Attribution Noncommercial License which permits any noncommercial use, distribution, and reproduction in any medium, provided the original author(s) and source are credited.

\section{References}

1. Arnold, V.I.: Geometrical methods in the theory of ordinary differential equations. Springer, New York (1993)

2. Bobieński, M., Żołądek, H.: The 3-dimensional generalized Lotka-Volterra systems. J. Ergod. Theory Dyn. Syst. 25, 759-791 (2005)

3. Gołaszewski, Ł.J., Sławiński, P., Żołądek, H.: Limit cycles in 3D Lotka-Volterra systems appearing after perturbation of Hopf center. Int. J. Bif. Chaos 18(12), 3647-3656 (2008)

4. Hirsch, M.: Systems of differential equations which are competitive or cooperative: III. Competing species. Nonlinearity 1, 51-71 (1988)

5. Hofbauer, J., Sigmund, K.: Evolutionary game dynamics. Bull. Am. Math. Soc. 40(4), 479-519 (2003)

6. Lotka, A.J.: Elements of mathematical biology. Dover Publications Inc., New York (1958)

7. Lubowiecki, P., Żołądek, H.: Invariant torus in 3D Lotka-Volterra systems. The non-degeneracy condition. Int. J. Bif. Chaos (in press)

8. Volterra, V.: Leçons sur la théorie mathématique de la lutte pour la vie. Gauthier-Villars, Paris (1931)

9. Zeeman, E.C., Zeeman, M.L.: From local to global behavior in competitive Lotka-Volterra systems. Trans. Am. Math. Soc. 355(2), 713-734 (2002)

10. Żołądek, H.: Quadratic systems with center and their perturbations. J. Diff. Equ. 109, 223-273 (1994) 